\title{
Can the Serum Level of Myostatin be Considered as an Informative Factor for Cachexia Prevention in Patients with Medullary Thyroid Cancer?
}

\author{
Mehdi Hedayati ${ }^{1 *}$, Zahra Nozhat ${ }^{1,2}$, Masoomeh Hannani ${ }^{1}$
}

\begin{abstract}
Thyroid cancer, the most common endocrine neoplasia, consists of four main types of carcinomas: papillary, follicular, and anaplastic, all with thyroid follicular origin, and medullary thyroid cancer (MTC) related to para-follicular cells. Cronic diseases such as diverse cancers may be associated with cachexia, especially at advanced stage. Cancer-induced cachexia is associated with diminished quality of life, functional performance, reduced response to antitumor therapy, and increased morbidity and mortality. Myostatin (Mst) is one of the outstanding molecules in the skeletal muscle loss process in cancer and it may be released by both skeletal muscle and cachexia-inducing tumors. Recently changes in serum levels of Mst have been identified as an important factor of cancer-induced cachexia. The goal of this study was to assessserum Mst levels in MTC patients. In this descriptive and case-control study, 90 participants were selected, comprising 45 MTC patients (20 males, $29 \pm 13.9$ years, 25 females, 29 \pm 14.5 years) and 45 control individuals (25 males, $23.1 \pm 11.6$ years, 20 females, $31.5 \pm 14.4$ years). Serum Mst was determined using an ELISA kit and body mass index (BMI) was calculated by weight and height measurements. The Kolmogorov Simonov test showed a normal distribution for log transformed Mst serum levels in both case and control groups. Geometric means were 5.9 and 8.2 $\mathrm{ng} / \mathrm{ml}$ respectively, and a significant difference was found according to the independent t-test results $(\mathrm{P}<0.01)$. There was also a significant difference mean of Mst between females in control and MTC groups, but not for the males. Pearson correlation test showed no correlation between age and BMI with Mst serum levels. The findings of this study support the hypothesis that Mst serum levels may have a potential ability for early diagnosis of cachexia in MTC patients, especially in females.
\end{abstract}

Keywords: Medullary thyroid cancer - cancer cachexia - myostatin - gender

Asian Pac J Cancer Prev, 17, Cancer Control in Western Asia Special Issue, 119-123

\section{Introduction}

Thyroid gland carcinoma is the most common endocrine system malignancy, including two main groups: 1) follicular thyroid cells derived cancers and 2) para-follicular thyroid cell derived carcinoma. The first one contains follicular (FTC), papillary (PTC) and anaplastic (ATC) thyroid cancers and the second one includes medullary thyroid cancer (MTC) (Wrtofsky et al., 2006; Cho et al., 2014). Roughly, 5\% of all human thyroid cancers are of MTC and it may take place in two forms of sporadic ( $75 \%$ of cases) and hereditary ( $25 \%$ in of cases) (Hedayati et al., 2006; Ceolin et al., 2012; Sheikholeslami et al., 2014). Three clinical forms of multiple endocrine neoplasia (MEN) type 2A, MEN 2B and familial MEN (FMEN) have been demonstrated for MTC manifesting
(Sheikholeslami et al., 2014). Hereditary pattern of MTC is autosomal dominant (Alvandi et al., 2011; Majidi et al., 2011; Ghazi et al., 2014) and in its hereditary form in addition to thyroid, parathyroid and adrenal glands are involved (Hedayati et al., 2011; Zarif Yeganeh et al., 2015a; Zarif Yeganeh et al., 2015b). Some chronic diseases such as diverse cancers are associated with cachexia, especially at the advanced stages of diseases. The word cachexia has been driven from two Greek words kakos and hexia meaning "bad condition" and has been demonstrated as a key sign in various malignancies (Donohoe et al., 2011). It is a complex and multifactorial syndrome characterized by: 1) unintended body weight loss due to loss of skeletal muscle with or without fat mass wasting, 2) anorexia, 3) inflammation, 4) insulin resistance, 5) increased 
muscle protein breakdown, and 6) metabolic alterations in the central nervous and immune systems. In spite of appropriate nutritional intake, it is roughly an irreversible condition showing resistance to conventional nutritional support (Donohoe et al., 2011; Baracos et al., 2013; Loumaye et al., 2015). Depending on the tumor type, around $50-80 \%$ of cancer patients are affected by skeletal muscle and adipose tissue atrophy and at least $20 \%$ of them died of cachexia (Tisdale et al., 2009; Shum et al., 2012). Cancer-induced cachexia is associated with diminished quality of life, functional performance, reduced response to antitumor therapy, and increased morbidity and mortality (Elkina et al., 2011; Penet et al., 2013; Smiyh et al., 2013). Cancer-induced cachexia may be manifested before other signs and symptoms of cancer and in the early stages of tumor growth; therefore, on the one hand, its earliest manifestation; on the other hand, its role in the death of cancer patients have made cancer cachexia as an important diagnostic factor whose early detection and control might result in the increase of quality of life and prolonged survival in affected patients (Kern et al., 1988). Nowadays, an increasing interest in therapeutic interventions has emerged with the aim to prevent, postpone or treat cancer-related body weight loss (Smith et al., 2013). However, there are many ambiguous aspects in the precise understanding of molecular mechanisms of cachexia, and its molecular basis has not been characterized completely yet (Evans et al., 2008; Loumaye et al., 2015). Recently new strategies of cachexia therapy have targeted distinct molecules such as the skeletal muscle androgen receptor, Mst, ghrelin, interleukin 6, and interleukin 1 (Baracos 2013). Mst is one of the outstanding molecules in skeletal muscle loss process in cancer and it may be released by both skeletal muscles and cachexia-inducing tumors (Argilés et al., 2014). Mst, a member of tumour growth factor $\beta$ (TGF- $\beta$ ) family, also known as growth/differentiation factor- 8 (GDF-8), has been introduced as a strong negative regulator of skeletal muscle growth (McPherron et al., 1997; Breibart et al., 2013). In the present study, in order to diminish the subsequent adverse effects of cachexia, we assumed a correlation between the manifested cachexia in MTC patients and their Mst serum levels. If there is a significant relationship between MTC-induced cachexia and serum levels of Mst, the use of Mst inhibitors will be promising to reduce the skeletal muscle wasting and fat mass loss in the case of MTC patients.

\section{Materials and Methods}

\section{Patients and sample preparation}

The present study was performed in the Cellular and Molecular Research Center (CMRC) Research Institute for Endocrine Sciences, Shahid Beheshti University of Medical Science. For the purpose of this investigation, 90 subjects were selected among which 45 were MTC patients (20 male $29 \pm 13.9$ years, 25 female $29 \pm 14.5$ years) who were referred from different health centers to the CMRC, and 45 volunteer controls (25 male $23.08 \pm 11.6$ years, 20 female $31.5 \pm 14.4$ years) without any history of cancer and systemic disease participated in this study. Written informed consent was obtained from participants and under aseptic condition $2 \mathrm{ml}$ venous blood was collected and then the samples were incubated for $5 \mathrm{~min}$ at the room temperature in order for clot formation. By centrifuging (Centrifuge 5702R, Eppendorf AG, Hamburg, Germany) at $3000 \mathrm{rpm}$ for $10 \mathrm{~min}$, serum was separated from plasma and stored at $-80^{\circ} \mathrm{C}$ before analysis. The participants' body mass indexes (BMIs) were calculated after weight and height measurement.

\section{Myostatin assessment}

In order to measure the serum Mst in patients diagnosed with MTC and in the volunteer controls, we used an ELISA assay by Human

Myostatin, ELISA, CUSABIO BIOTECH, and Wuhan China Sensitivity: $0.31 \mathrm{ng} / \mathrm{ml}$.

Statistical analysis: The present study is a descriptive and case-control one. In order to design the study,

MedCalc software was applied to calculate the sample size. Continuous quantitative variable analysis and comparison of two means (two groups' means) were carried out by Kolmogorov-Smirnov and independent " $t$ " test respectively. Pearson correlation coefficients were determined between serum levels of Mst and BMI, and age in the both MTC and control groups.

\section{Results}

According to the obtained results from MedCalc software, the sample size was calculated 45 subjects for both case and control groups (overall 90 participants). The confidence interval (type I error, $\alpha=0.05$ ), study power (with type II error, $\beta=0.2$ ), mean variation and standard deviation were evaluated $95 \%, 80 \%, 1.2$ and 2 respectively. The Kolmogorov-Smirnov test demonstrated that the distribution of age and BMI as continuous quantitative variables were normal and it also showed that log transformed of Mst levels had a normal distribution in both MTC and control groups. In two participants (one of them from MTCs and the other from control group) the levels of Mst were far out of the range; therefore, the related data were set aside. In the Table1, the subjects' demographic, anthropometric and biochemical data were demonstrated. The geometric mean of Mst levels between MTC and control groups were compared by independent "t" test and statistical significance of the test considered $\mathrm{p}<0.05$. According to the results of independent " $\mathrm{t}$ " test, there was a significant difference between Mst concentration means in MTC and control groups. No significant statistical difference was observed between BMI and age means in MTC and control groups. Geometric mean of Mst in patient and control groups was 5.9 and $8.2 \mathrm{ng} / \mathrm{ml}$, respectively with $\mathrm{P}<0.01$, showing statistically significant difference between two groups (Figure1). On the other hand, a significant difference was found between the means of serum levels of Mst in female gender of control and MTC groups $(\mathrm{P}=0.2)$ but such difference was not observed between males in control and MTC groups $(\mathrm{P}=0.03)$. According to the Pearson correlation tests, no correlation was seen between age and 
Table1. Demographic, Anthropometric and Biochemical Data of Cases (MTC Patients and Controls)

\begin{tabular}{|c|c|c|c|c|c|c|c|}
\hline Variables & Unit & Subjects & Minimum & Maximum & Mean & Female & Male \\
\hline \multirow[t]{2}{*}{ Age } & year & control & 20.0 & 60.0 & 32.2 & $33.0 \pm 10.5$ & $31.1 \pm 9.6$ \\
\hline & & case & 20.0 & 59.0 & 33.3 & $35.6 \pm 12.6$ & $30.3 \pm 7.8$ \\
\hline \multirow[t]{2}{*}{ BMI } & $\mathrm{kg} / \mathrm{m} 2$ & control & 24.0 & 27.9 & 25.8 & $25.7 \pm 0.9$ & $26 \pm 1.4$ \\
\hline & & case & 24.1 & 28.9 & 26.2 & $26.4 \pm 1.2$ & $26.1 \pm 1.4$ \\
\hline \multirow[t]{2}{*}{ Myostatin } & $\mathrm{ng} / \mathrm{ml}$ & control & 3.1 & 18.5 & 8.2 & $9.8 \pm 5.3$ & $9 \pm 4.2$ \\
\hline & & case & 2.0 & 17.9 & 5.9 & $8.2 \pm 4.6$ & $6.1 \pm 4.3$ \\
\hline
\end{tabular}

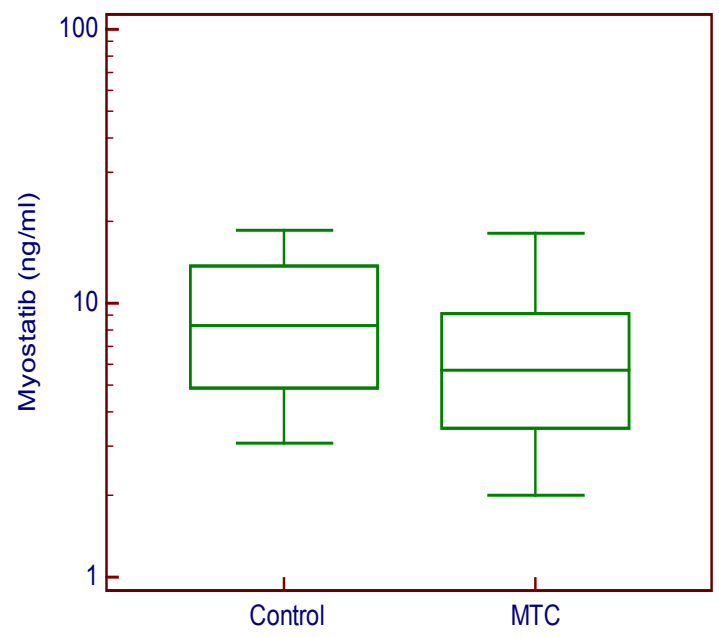

Figure 1. Independent t-test Showed a Significant Difference between Logarithmic Transformations of Mean Mst in MTC and Control Groups.
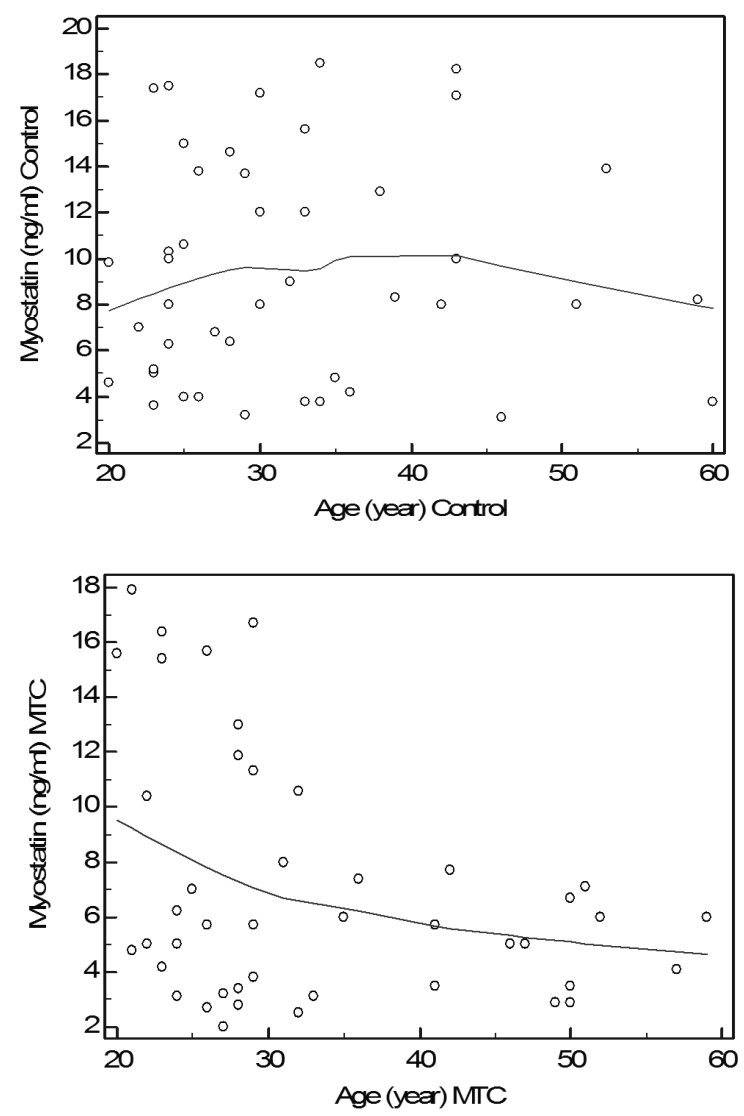

BMI with Mst serum levels (Figure2).

\section{Discussion}

In spite of the animal studies findings showing cancer-induced muscle wasting as a significant outcome of circulating proteins produced by the host and tumor cells, the effects have not been identified completely (Lokireddy et al., 2012). According to the recent findings emphasizing Mst critical role in the cancer cachexia, in the present study we assumed that the Mst serum levels in the affected patients by MTC will be higher in comparison with healthy controls. The results of this research support our hypothesis and we found a significant difference between serum Mst concentrations in two groups. This finding is consistent with the results of many different studies which examined Mst in cancer-induced cachexia in various cancers. In 2012, Lokireddy el al. introduced the Mst as a novel tumor factor inducing cancer cachexia. In order to identify tumoral factors involved in
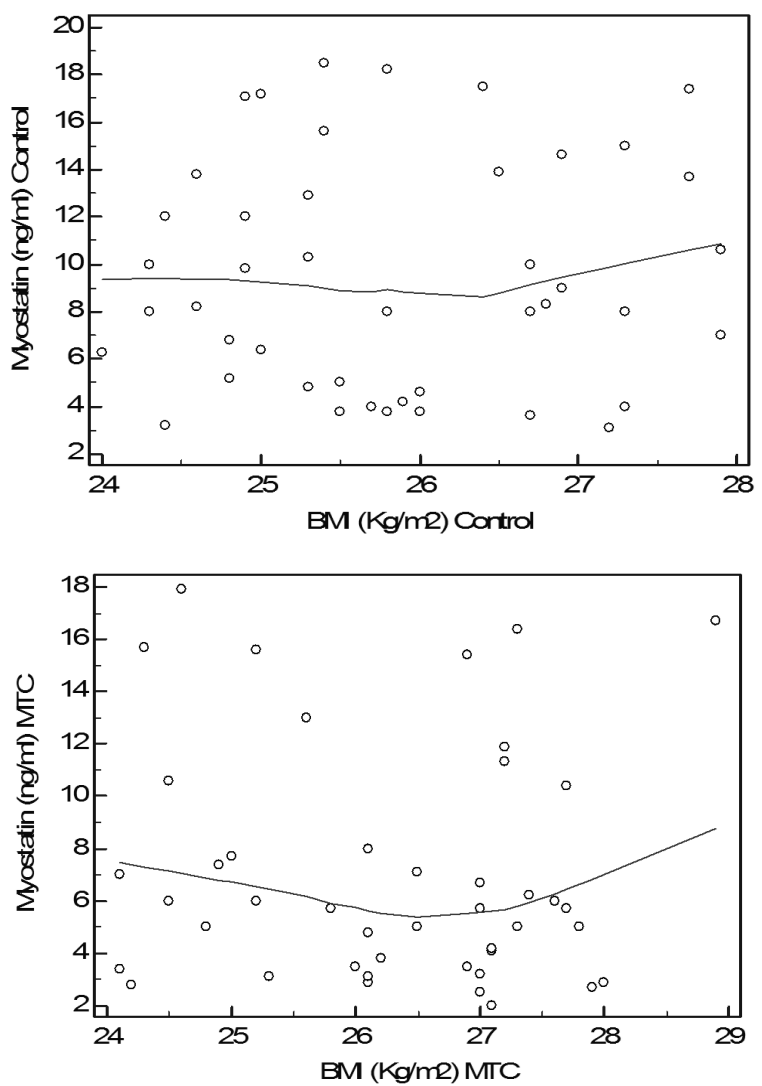

Figure2. Pearson Correlation Test Showed no Correlation between Age/Mst and BMI/Mst Levels in Both Control and MTC Groups 
cancer-induced cachexia, they investigated the secretome of C26, colon cancer cells. Abundant secretion of Mst was observed by $\mathrm{C} 26$ cell line. To confirm the Mst signaling during cachexia, differentiated $\mathrm{C} 2 \mathrm{C} 12$ myotubes were treated by the medium including $\mathrm{C} 26$ secretom and myotubular atrophy was observed following this treatment. They also reported the induction of autophagy-lysosome pathway and reduction of the number of mitochondria in myotubes when they were treated with C26 conditional medium (Lokireddy et al., 2012). Aversa et al studied Mst signaling in muscle samples obtained from gastric and lung cancer patients. The expression of Mst in patients who suffered from gastric cancer significantly increased but in lung cancer there was no difference between Mst levels in patients and controls. Such different expression of Mst was attributed to different patterns of molecular changes within the muscle in different cancer types (Aversa et al., 2012). In another study performed by Bonetto and Aversa's teamwork, a reduction in Mst mRNA expression in skeletal muscle of gastric cancer patients was reported. They found this result surprising and despite the ambiguous reasons they suggested that such different results may be due to some factors such as posttranslational modifications, disease stage and or muscle activation to maintain the homeostasis (Bonetto et al., 2103). We found very few studies investigated the relationship between thyroid disorders and Mst. Carneiro et al, demonstrated an increased levels of Mst expression in hyperthyroidism rats associated muscle wasting (Carneiro et al., 2008). To our knowledge no study was found to have investigated the relationship between Mst serum levels and thyroid cancer cachexia previously.

In this study, not only we found that there is statistically a significant difference between Mst serum levels in MTC and control groups, but also we observed a difference between this molecule concentrations among females in two groups. The latter difference can be attributed to lower muscle mass in the females, which can be affected by cancer condition sooner than males. Forasmuch as MTC has a low incidence, in this study cancer stage did not equal in all cases, and this can be considered as a limitation of the research.

In conclusion, this investigation has shown that serum levels of Mst can be considered as an early diagnosis biomarker for identifying cancer-induced cachexia in MTC patients. This study can be a starting point for more investigations in order to introduce Mst as a significant tumor factor inducing cancer cachexia.

\section{Acknowledgment}

This work was supported by the Cellular and Molecular Research Center (CMRC) Research Institute for Endocrine Sciences, and Shahid Beheshti University of Medical Science. The authors appreciate the kind cooperation of the endocrinologist, healthy volunteer participants and patients affected by MTC. They also give thanks to the CMRC.There is no conflict of interest to declare for any of the authors.

\section{References}

Alvandi E, Akrami SM, Chiani M, et al (2011). Molecular analysis of the RET proto-oncogene key exons in patients with medullary thyroid carcinoma: a comprehensive study of the Iranian population. Thyroid, 21, 373-82.

Argilés JM, Busquets S, Stemmler B, López-Soriano FJ, (2014). Cancer cachexia: understanding the molecular basis. Nat Rev Cancer, 14, 754-62.

Aversa Z, Bonetto A, Penna F, et al (2012). Changes in myostatin signaling in non-weight-losing cancer patients. Ann Surg Oncol, 19, 1350-6.

Baracos VE, (2013). Clinical Trials of Cancer Cachexia Therapy, Now and Hereafter. J Clin Oncol, 31, 1257-8.

Bonetto A, Penna F, Aversa Z, et al (2013). Early changes of muscle insulin-like growth factor-1 and myostatin gene expression in gastric cancer patients. Muscle Nerve, $\mathbf{4 8 ,}$ 387-92.

Breitbart A, Scharf GM, Duncker D et al, (2013). Highly specific detection of myostatin prodomain by an immunoradiometric sandwich assay in serum of healthy individuals and patients. Plos One, 8, e80454-9.

Carneiro I, Castro-Piedras I, Muñoz A, et al (2008). Hypothyroidism is associated with increased myostatin expression in rats. $J$ Endocrinol Invest, 31, 773-8.

Ceolin L, Siqueira DR, Romitti M, Ferreira CV, Maia AL (2012). Molecular basis of medullary thyroid carcinoma: the role of RET polymorphisms. Int J Mol Sci, 13, 221-39.

Cho YA, Kong SY, Shin A, et al (2014). Biomarkers of thyroid function and autoimmunity for predicting high-risk groups of thyroid cancer: a nested case-control study. BMC cancer, 14, 873.

Donohoe CL, Ryan AM, Reynolds JV, (2011). Cancer cachexia: mechanisms and clinical implications. Gastroenterol Res Pract, 2011,60143-4

Elkina Y, von Haehling S, Anker SD, Springer J, (2011). The role of myostatin in muscle wasting: an overview. $J$ Cachexia Sarcopenia Muscle, 2, 143-51.

Evans WJ, Morley JE, Argilés J, et al (2008). Cachexia: a new definition. Clin Nutr, 27, 793-9.

Ghazi AA, Bagheri M, Tabibi A, Sarvghadi F, et al (2014). Multiple endocrine neoplasia type 2A in an Iranian family: clinical and genetic studies. Arch Iran Med, 17, 378-82.

Hedayati M, Nabipour I, Rezaei-Ghaleh N, Azizi F (2006). Germline RET mutations in exons 10 and 11: an Iranian survey of 57 medullary thyroid carcinoma cases. Med $J$ Malaysia, 61, 564-9.

Hedayati M, Zarif Yeganeh M, SheikholEslami S, et al (2011). Predominant RET germline mutations in exons 10, 11, and 16 in Iranian patients with hereditary medullary thyroid carcinoma. J Thyroid Res, 2011, 26424-8.

Kern KA, Norton JA, (1988). Cancer cachexia. J Parenter Enteral Nutr, 12, 286-98.

Lokireddy S, Wijesoma IW, Bonala S, et al, (2012). Myostatin is a novel tumoral factor that induces cancer cachexia. Biochem J, 446, 23-36.

Loumaye A, de Barsy M, Nachit M, et al (2015). Role of Activin A and Myostatin in human cancer cachexia. J Clin Endocrinol Metab, 100, 2030-8.

Majidi M, Haghpanah V, Hedayati M, et al (2011). A family presenting with multiple endocrine neoplasia type $2 \mathrm{~B}$ : A case report. J Med Case Rep, 5, 587-92.

McPherron AC, Lawler AM, Lee SJ, (1997). Regulation of skeletal muscle mass in mice by a new TGF-beta superfamily 
member. Nature, 387, 83-90.

Penet MF, Winnard PT Jr, Jacobs MA, et al (2011). Understanding cancer-induced cachexia: imaging the flame and its fuel. Curr Opin Support Palliat Care, 5, 327-33.

Sheikholeslami S, Zarif Yeganeh M, Hoghooghi Rad L, Golab Ghadaksaz H, Hedayati M (2014). Haplotype frequency of G691S/S904S in the RET proto-onco-gene in patients with medullary thyroid carcinoma. Iran J Public Health, 43, 235-40.

Shum AM, Polly P, (2012). Cancer cachexia: molecular targets and pathways for diagnosis and drug intervention. Endocr Metab Immune Disord Drug Targets, 12, 247-59.

Smith RC, Lin BK, (2013). Myostatin inhibitors as therapies for muscle wasting associated with cancer and other disorders. Curr Opin Support Palliat Care, 7, 352-60.

Tisdale MJ, (2009). Mechanisms of cancer cachexia. Physiol Rev, 89, 381-410.

Zarif Yeganeh M, Sheikholeslami S, Dehbashi Behbahani G, Farashi S, Hedayati M (2105). Skewed mutational spectrum of RET proto-oncogene Exon10 in Iranian patients with medullary thyroid carcinoma. Tumour Biol, 36, 5225-31.

Zarif-Yeganeh M, Sheikholeslami S, Dehbashi-Behbahani G, et al (2015). Point Mutations in RET Proto-Oncogene Exon 10 in Patients with Medullary Thyroid Carcinoma. J Kerman Univ Med Sci, 22, 249-60. 